\title{
A CHARACTERIZATION OF FOUR-DIMENSIONAL EINSTEIN MANIFOLDS
}

\author{
YI-CHING YEN
}

AbSTRACT. It is shown that for any $P$ on a four-dimensional Einstein manifold $M$, there exists an orthonormal basis $\left\{Y_{i}\right\}$ in $T_{p}(M)$ such that all the sectional curvatures $K\left(Y_{i}, Y_{j}\right)$ are equal.

1. Let $M$ be an $n$-dimensional Riemannian manifold with inner product $g$. A covariant curvature tensor with respect to $g$ at every point $P \in M$ is [1] a multilinear map $R: T_{p}(M) \times T_{p}(M) \times T_{p}(M) \times T_{p}(M) \rightarrow R$, or

$$
R\left(X_{i}, X_{j}, X_{k}, X_{l}\right)=R_{i j k l}
$$

for an orthonormal basis $\left\{x_{i}\right\}$ in the tangent space $T_{p}(M)$, where the curvature tensor satisfies the usual identities

$$
\begin{gathered}
R_{i j k l}=-R_{j i k l}=-R_{i j l k}=R_{k l i j}, \\
R_{i j k l}+R_{i k l j}+R_{i l j k}=0 .
\end{gathered}
$$

We define the sectional curvature $K$ as follows: If $\sigma=\{X, Y\}$ is a 2plane $\subseteq T_{p}(M)$, then

$$
K(X, Y)=\frac{R(X, Y, Y, X)}{\langle X, X\rangle\langle Y, Y\rangle-\langle X, Y\rangle^{2}},
$$

where $\langle X, Y\rangle=g(X, Y)$ is the inner product of $X, Y$. In case $X_{i}, X_{j}$ are orthonormal,

$$
K\left(X_{i}, X_{j}\right)=R\left(X_{i}, X_{j}, X_{j} X_{i}\right)=-R_{i j i j} .
$$

A Ricci tensor is a bilinear map defined by

$$
\operatorname{Ric}(Y, Z)=\sum_{i=1}^{n} R\left(X_{i}, Y, Z, X_{i}\right),
$$

$\left\{X_{i}\right\}$ being an orthonormal basis of $T_{p}(M)$.

An Einstein manifold $M$ is a Riemannian manifold with metric $g$ satisfying

$$
\operatorname{Ric}(Y, Z)=\operatorname{ag}(Y, Z) \text {. }
$$

Received by the editors March 1, 1972 and, in revised form, January 3, 1973. AMS (MOS) subject classifications (1970). Primary 53C25; Secondary 53C25.

(c) American Mathematical Society 1973 
where $a$ is a constant. By taking an orthonormal basis $\left\{X_{i}\right\}$ in $T_{p}(M)$ at a point $p \in M$, we have $\operatorname{Ric}\left(X_{j}, X_{k}\right)=a g\left(X_{j}, X_{k}\right)=a \delta_{j k}$ for an Einstein manifold, where $\delta_{j k}$ is the Kronecker delta.

2. Before discussing our theorem, we introduce some lemmas as follows; the first two are well known.

Lemma 1. The sectional curvature of a 2-plane $\sigma$ is independent of the choice of basis vectors of $\sigma$.

LEMMA 2 (SCHUR). The sectional curvature is a constant $K_{0}$ if and only if

$$
R_{i j k l}=K_{0}\left(g_{i l} g_{j k}-g_{i k} g_{j l}\right) .
$$

Lemma 3 [1]. Let $\left\{X_{1}, \cdots, X_{4}\right\}$ be an orthonormal basis of the tangent space $T_{p}$ of a four-dimensional Einstein manifold, and denote $K\left(X_{i}, X_{j}\right)$ by $K_{i j}$. Then

$$
K_{12}=K_{34}, \quad K_{13}=K_{24}, \quad K_{14}=K_{23} .
$$

Proof. From (1), (6), (7) it follows that $\operatorname{Ric}\left(X_{j}, X_{k}\right)=\sum_{i=1}^{4} R_{i j k i}=a \delta_{j k}$, which becomes, for $j=k=1, R_{2112}+R_{3113}+R_{4114}=a$, or

$$
K_{12}+K_{13}+K_{14}=a \text {. }
$$

Similarly, we have

Noting $K_{i j}=K_{j i}$ from the definition of the sectional curvature, and adding (10), (11) together, we obtain

$$
K_{12}+K_{13}+K_{14}+K_{23}+K_{24}+K_{34}=2 a \text {. }
$$

Again, from (10) and (11) it follows that

$$
\left(K_{12}+K_{13}+K_{14}\right)+\left(K_{12}+K_{23}+K_{24}\right)=2 a,
$$

which, together with (12), implies $K_{12}=K_{34}$.

Similar computations yield the other two equalities of (9).

Lemma 4 [1]. There exists an orthonormal basis $\left\{X_{i}\right\}$ of $T_{p}(M)$ of a fourdimensional Einstein manifold $M$ such that all components, except $K\left(X_{j}, X_{k}\right)$, $R_{1234}, R_{1324}, R_{1423}$, of the curvature tensor are zero.

PROof. The possible nonzero components of $R_{i j k l}(1 \leqq i, j, k, l \leqq 4)$ are $K_{j k}, R_{i k j k}(j, k$ distinct and $\neq i)$, and $R_{i j k l}$ (with distinct $\left.i, j, k, l\right)$. We shall show that all $R_{i k j k}$ are zero.

Let the 2-plane $\sigma\left(X_{1}, X_{2}\right) \subseteq T_{p}(M)$ be so chosen that $K\left(X_{1}, X_{2}\right)$ is the extremal value of the sectional curvatures at $P \in M$. This is possible since 
orthonormal bases $\{X, Y\}$ form a closed set in $T_{p}(M)$ topologically and $K(X, Y)$ is a continuous function defined there.

Let the 2-plane $\zeta\left(X_{3}, X_{4}\right) \subseteq T_{p}(M)$ be the orthogonal complement of $\sigma\left(X_{1}, X_{2}\right) ; X_{3}$ and $X_{4}$ can be arbitrarily chosen in this 2-plane. For any $Y \in \sigma\left(X_{1}, X_{2}\right), Z \in \zeta\left(X_{3}, X_{4}\right)$, let $X_{1}, X_{3}$ be the vectors which give extremal values of $K(Y, Z)$. Then $X_{2}, X_{4}$ are determined naturally. Since $K\left(X_{1}, X_{2}\right)$ is extremal,

$$
\begin{array}{ll}
K\left(X_{1}, X_{2} \cos \theta+X_{3} \sin \theta\right), & K\left(X_{1}, X_{2} \cos \theta+X_{4} \sin \theta\right), \\
K\left(X_{2}, X_{1} \cos \theta+X_{3} \sin \theta\right), & K\left(X_{2}, X_{1} \cos \theta+X_{4} \sin \theta\right)
\end{array}
$$

are stationary at $\theta=0$, e.g., by setting

$f(\theta)=K\left(X_{1}, X_{2} \cos \theta+X_{3} \sin \theta\right)=K_{12} \cos ^{2} \theta-R_{1213} \sin 2 \theta+K_{13} \sin ^{2} \theta$, we have $f^{\prime}(\theta)=-K_{12} \sin 2 \theta-2 R_{1213} \cos 2 \theta+K_{13} \sin 2 \theta$, which is zero at $\theta=0$, so that $f^{\prime}(0)=-2 R_{1213}=0$. Thus for the four given functions of $\theta$ given in (14), we obtain

$$
R_{1213}+R_{1214}=R_{1223}=R_{1224}=0 .
$$

Similarly, since $K\left(X_{1}, X_{3}\right)$ is extremal,

$$
K\left(X_{1}, X_{3} \cos \theta+X_{4} \sin \theta\right), \quad K\left(X_{3}, X_{1} \cos \theta+X_{2} \sin \theta\right)
$$

take stationary values at $\theta=0$, and therefore

$$
R_{1314}=R_{1323}=0 \text {. }
$$

On the other hand, $\operatorname{Ric}\left(X_{j}, X_{k}\right)=\sum_{i=1}^{4} R_{i j k l}=a \delta_{j k}$, which becomes for $j=2$ and $k=3, R_{1213}+R_{2434}=0$, so that

$$
R_{2434}=0 \text {, }
$$

since $R_{1213}=0$. By similar computations we obtain

$$
R_{2334}=R_{1434}=R_{1334}=R_{2324}=R_{1424}=0 .
$$

By (2), (15), (17), (18) and (19), the possible nonzero $R_{i j k l}$ are thus $K_{j k}$ $(1 \leqq j<k \leqq 4), R_{1234}, R_{1324}$, and $R_{1423}$ for such basis $\left\{X_{i}\right\}$.

3. THEOREM. There exists an orthonormal basis $\left\{Y_{i}\right\}$ of $T_{p}(M)$ of a fourdimensional Einstein manifold $M$ such that all sectional curvatures $K\left(Y_{j}, Y_{k}\right)$ are equal.

Proof. It should be remarked that if $K$ is constant, every orthonormal basis $\left\{Y_{i}\right\}$ of $T_{p}(M)$ is the required one.

For the general case, we choose $\left\{X_{i}\right\}$ to be an orthonormal basis as stated in Lemma 4 , so that

$$
R_{i k j k}=0 \quad(i \neq j) .
$$


In view of Lemma 3, we need only to show that $K\left(Y_{1}, Y_{2}\right)=K\left(Y_{2}, Y_{3}\right)=$ $K\left(Y_{3}, Y_{1}\right)$ for the required basis $\left\{Y_{i}\right\}$. To this end we let $K_{12} \geqq K_{23} \geqq K_{31}$. Consider an orthogonal transformation of $O(4)$, which keeps $X_{4}$ fixed and rotates about the triple $\left\{X_{1}, X_{2}, X_{3}\right\}$. Let this transformation be of the form $Y_{i}=T x_{i}, i=1, \cdots, 4$. Since $Y_{4}=X_{4}, T=\left(\begin{array}{c}A \\ 0 \\ 0\end{array}\right)$ with $Y_{i}=A X_{i}, i=1,2,3$, and $A=\left(\alpha_{j k}\right)$ is an orthogonal matrix. Let $\operatorname{Adj} A=\left(\beta_{j k}\right), K_{12}=K_{23}+\varepsilon_{1}$, $\varepsilon_{1} \geqq 0 ; K_{13}=K_{23}-\varepsilon_{2}, \varepsilon_{2} \geqq 0$; and $R_{j k}=K\left(Y_{j}, Y_{k}\right)$. Then by (20),

$$
\begin{aligned}
R_{12}= & \left(\alpha_{11} \alpha_{22}-\alpha_{12} \alpha_{21}\right)^{2} K_{12}+\left(\alpha_{12} \alpha_{23}-\alpha_{13} \alpha_{22}\right)^{2} K_{23} \\
& +\left(\alpha_{13} \alpha_{21}-\alpha_{11} \alpha_{23}\right)^{2} K_{13} \\
= & \beta_{33}^{2} K_{12}+\beta_{13}^{2} K_{23}+\beta_{23}^{2} K_{13}, \quad\left(\text { since } \sum_{j=1}^{3} \beta_{j 3}^{2}=1\right) \\
= & K_{23}+\beta_{33}^{2} \varepsilon_{1}-\beta_{23}^{2} \varepsilon_{2} .
\end{aligned}
$$

Similarly,

$$
R_{23}=K_{23}+\beta_{31}^{2} \varepsilon_{1}-\beta_{21}^{2} \varepsilon_{2}, \quad \bar{K}_{31}=K_{23}+\beta_{32}^{2} \varepsilon_{1}-\beta_{22}^{2} \varepsilon_{2} .
$$

Thus $R_{12}=R_{23}=R_{31}$ provided that

$$
\begin{aligned}
\beta_{3 k}^{2} \varepsilon_{1}-\beta_{2 k}^{2} \varepsilon_{2} & =\frac{1}{3}\left[\varepsilon_{1}\left(\beta_{31}^{2}+\beta_{32}^{2}+\beta_{33}^{2}\right)-\varepsilon_{2}\left(\beta_{23}^{2}+\beta_{21}^{2}+\beta_{22}^{2}\right)\right] \\
& =\frac{1}{3}\left(\varepsilon_{1}-\varepsilon_{2}\right), \quad k=1,2,3
\end{aligned}
$$

Among the three equations of (23), only two of them are linearly independent due to the facts that $\sum_{k=1}^{3} \beta_{j k}^{2}=1, j=2,3$. Since the orthogonality of the matrix $\left(\beta_{j k}\right)$ is given by

$$
\sum_{k=1}^{3} \beta_{j k} \beta_{l k}=\delta_{j l}, \quad j, l=1,2,3,
$$

$\left(\beta_{j k}\right)$ can be found by the two independent equations of (23) and the six equations of (24) indicated as follows:

$\left(\beta_{11}, \beta_{21}, \beta_{31}\right)$ can be found arbitrarily by the equations

$$
\varepsilon_{1} \beta_{31}^{2}-\varepsilon_{2} \beta_{21}^{2}=\frac{1}{3}\left(\varepsilon_{1}-\varepsilon_{2}\right), \quad \beta_{11}^{2}+\beta_{21}^{2}+\beta_{31}^{2}=1,
$$

and for one such solution, $\left(\beta_{12}, \beta_{22}, \beta_{32}\right)$ is determined by

$$
\begin{aligned}
\beta_{11} \beta_{12}+\beta_{21} \beta_{22}+\beta_{31} \beta_{32} & =0, \\
\varepsilon_{1} \beta_{32}^{2}-\varepsilon_{2} \beta_{22}^{2} & =\frac{1}{3}\left(\varepsilon_{1}-\varepsilon_{2}\right), \\
\beta_{12}^{2}+\beta_{22}^{2}+\beta_{32}^{2} & =1 .
\end{aligned}
$$


Then $\left(\beta_{13}, \beta_{23}, \beta_{33}\right)$ follows from

$$
\begin{array}{r}
\beta_{11} \beta_{13}+\beta_{21} \beta_{23}+\beta_{31} \beta_{33}=0, \\
\beta_{12} \beta_{13}+\beta_{22} \beta_{23}+\beta_{32} \beta_{33}=0, \\
\beta_{13}^{2}+\beta_{23}^{2}+\beta_{33}^{2}=1 .
\end{array}
$$

For such orthogonal matrix $\operatorname{Adj} A=\left(\beta_{j k}\right), A=\left(\alpha_{j k}\right)=(\operatorname{Adj} A)^{-1}|A|=$ $(\operatorname{Adj} A)^{t}=\left(\beta_{k j}\right)$ is thus obtained; e.g., for $\varepsilon_{2}=0$ (i.e., $K_{12}>K_{23}=K_{31}$ ), or $\varepsilon_{1}=\varepsilon_{2}$ (i.e., $K_{23}=\frac{1}{2}\left(K_{12}+K_{31}\right)$ ), we have respectively

or

$$
A=\left(\begin{array}{ccc}
\frac{1}{\sqrt{ } 2} & \frac{1}{\sqrt{ } 6} & \frac{1}{\sqrt{ } 3} \\
-\frac{1}{\sqrt{ } 2} & \frac{1}{\sqrt{ } 6} & \frac{1}{\sqrt{ } 3} \\
0 & -\frac{2}{\sqrt{ } 6} & \frac{1}{\sqrt{ } 3}
\end{array}\right)
$$

$$
A=\left(\begin{array}{ccc}
1 & 0 & 0 \\
0 & \frac{1}{\sqrt{ } 2} & \frac{1}{\sqrt{ } 2} \\
0 & -\frac{1}{\sqrt{ } 2} & \frac{1}{\sqrt{ } 2}
\end{array}\right)
$$

by the above method.

REMARK. By Lemma 1, the orthonormal basis $\left\{Y_{i}\right\}$ may be replaced by an orthogonal basis $\left\{\bar{Y}_{i}\right\}$, where $\bar{Y}_{i}$ are parallel to $Y_{i}$.

\section{REFERENCE}

1. S. Tachibana, Riemannian geometry, Modern Math. Series, 1968. (Japanese)

Department of Mathematics, Fu-Jen University, Taipei, Taiwan, Republic of CHINA 